\title{
CONSIDERAÇÕES ANESTÉSICAS NA CIRURGIA LAPAROSCÓPICA
}

\author{
Anesthetic considerations in laparoscopic surgery
}

\author{
Carla Maria Olivetti CORRÊA, Maria José Nascimento BRANDÃO, \\ Eunice Sizue HIRATA, Artur UDELSMANN
}

ABCDDV/611

Corrêa CMO, Brandão MJN, Hirata ES, Udelsmann A. Considerações anestésicas na cirurgia laparoscópica. ABCD Arq Bras Cir Dig 2008;21(3):136-8

RESUMO - Introdução - Laparoscopias experimentais datam do início do século XX, mas somente a partir de 1960 é que começaram a ser experimentadas em humanos. Em 1985, na Alemanha, foi realizada a primeira colecistectomia e desde então a técnica teve grande divulgação e aceitação. O pneumoperitônio tem impacto em vários sistemas e tal deve ser considerado por ocasião da anestesia. Objetivo - Realizar revisão bibliográfica das alterações fisiológicas de interesse para o anestesiologista durante a cirurgia digestiva videolaparoscópica. Métodos - Foi realizada pesquisa bibliográfica em livros de anestesia disponíveis na biblioteca da Faculdade de Ciências Médicas da UNICAMP e em artigos de interesse publicados a partir de 1990 no endereço eletrônico PubMed http://www.ncbi.nlm.nih.gov/pubmed/ usando-se os descritores: anestesia, cirurgia, laparoscopia. Vinte e seis artigos foram revisados e um capítulo de livro. Conclusões - A cirurgia digestiva laparoscópica foi grande avanço em razão do menor impacto pós-operatório com diminuição da dor pós-operatória, alta hospitalar e retorno às atividades laborativas precoces. Entretanto suas particularidades não devem ser negligenciadas e cuidados particulares devem ser tomados pelo anestesiologista em indivíduos hipovolêmicos e cardiopatas. A capnometria durante a intervenção é imperativa.

DESCRITORES - Anestesia. Cirurgia. Laparoscopia.

\section{INTRODUÇÃO}

A idéia de visualizar e intervir em órgãos internos sem a necessidade de laparotomia é antiga. Já em 1901, Kelling, em Berlim, realizou a primeira laparoscopia experimental em cães, inclusive criando um pneumoperitônio com ar atmosférico ${ }^{15}$. Em 1929 Kalk, também na Alemanha, desenvolveu sistema de lentes oblíquas e preconizou uma punção separada para o pneumoperitônio ampliando os movimentos do operador ${ }^{14}$. Kurt Semm, considerado o pai da cirurgia laparoscópica, iniciou seus estudos na década de 1960 em Munique, onde desenvolveu um insuflador automático para a manutenção do pneumoperitônio, um dispositivo para monitorizar a pressão intra-abdominal e, ao usar $\mathrm{CO}^{2}$ para a insuflação, aumentou muito a segurança da técnica. Seus esforços, porém, não foram bem recebidos por seus pares na época. Sua persistência, no entanto, o fez continuar e tornar-se pioneiro na cirurgia laparoscópica realizando procedimentos como lise de aderências, biópsias, estadiamento de tumores e apendicectomias ${ }^{13}$.

Concomitantemente, um conjunto de inovações foram

Trabalho realizado no Serviço de Anestesia do Hospital das Clínicas da UNICAMP e Departamento de Anestesiologia da Faculdade de Ciências Médicas da UNICAMP, Campinas, SP, Brasil

Correspondência: Artur Udelsmann, e-mail: audelsmann@yahoo.com.br surgindo como o advento de microcâmeras, a transmissão de imagens nítidas por fibras ópticas e a produção de luz fria tornando segura a iluminação de cavidades orgânicas, entre outras, que proporcionaram base tecnológica suficiente para a videocirurgia digestiva se desenvolver. Influenciado por Semm, Erich Mühe, outro cirurgião alemão, tendo recebido um laparoscópio em seu serviço, realizou a primeira colecistectomia laparoscópica em setembro de 1985, cinco anos depois da primeira apendicectomia de Semm.

Mühe também teve de enfrentar rejeição ao seu trabalho porque, coincidentemente, nessa época houve grande melhora dos cuidados nas unidades de terapia intensiva e o desenvolvimento de novas drogas de anestesia que propiciaram a realização de grandes operações oncológicas e transplantes de órgãos, nos quais estava mais focada a política de saúde na Alemanha ${ }^{17}$. Além disso, também tentava-se tratamento não cirúrgico para os cálculos biliares, com drogas que os dissolvessem ou processo semelhante à litotripsia extracorpórea dos cálculos urinários, o que desestimulava a prática. Mas a técnica foi assim mesmo se expandindo e em 1987, sem saber dos trabalhos de Mühe, Mouret, na França, também realizou uma colecistectomia laparoscópica ${ }^{12,16}$.

No entanto, a consagração do método deu-se sem dúvida na América do Norte e em 1992, em reunião de consenso do Instituto Nacional de Saúde, considerou-se a colecistectomia laparoscópica o tratamento de escolha para a litíase biliar ${ }^{19}$. 
Portanto, após a resistência inicial, a videocirurgia difundiu-se com rapidez surpreendente e passou a ser indicada mesmo para procedimentos de maior porte realizados em pacientes muitas vezes com estado físico mais comprometido. A videocirurgia proporciona vantagens em relação à cirurgia convencional que são: menores incisões, menor incidência de íleo paralítico, menor comprometimento da função respiratória preservando a atividade diafragmática, menor formação de aderências, dor quase sempre menos intensa com deambulação e alta mais precoce ${ }^{2,9}$. Por essas razões é frequentemente chamada na literatura médica de cirurgia minimamente invasiva, quase que subestimando as alterações fisiológicas decorrentes do pneumoperitônio que podem chegar a ser de grande repercussão sistêmica.

\section{Alterações fisiológicas do pneumoperitônio}

$\mathrm{O}$ aumento da pressão intra-abdominal (PIA) decorrente do pneumoperitônio causa alterações significativas em função da pressão utilizada para insuflar, a duração do procedimento, o estado funcional cardiocirculatório, o posicionamento do paciente em céfalo-aclive ou céfalo-declive e sua volemia. O aumento da PIA exerce efeito mecânico de compressão sobre a vasculatura abdominal no território arterial, venoso e capilar.

A compressão sobre a aorta aumenta a resistência vascular periférica (RVP) e a pós-carga. A compressão sobre as veias, sistema de alta complacência e baixa resistência sofre influência importante do pneumoperitônio, com redução do retorno venoso e da pré-carga além de sequestro de sangue nas extremidades inferiore ${ }^{20}$. $\mathrm{O}$ aumento da pós-carga, a diminuição da pré-carga tendem a reduzir o volume sistólico e consequentemente o débito cardíaco.

Este fato pode ser agravado ou atenuado pelo estado de hidratação prévio do paciente, pelo posicionamento em céfalo-aclive (agravado) ou céfalo-declive (atenuado) e pelo valor da pressão de insuflação. Muitos autores consideram a pressão de insuflação máxima segura de $15 \mathrm{mmHg}$ em adultos e em crianças $10 \mathrm{mmHg}$. Estas últimas geralmente têm maior complacência abdominal e não é nelas necessária pressão maior para se obter pneumoperitônio que permita boa visualização ${ }^{7,27}$. Dependendo da combinação desses fatores, a redução do débito cardíaco pode no entanto ser drástica, chegando a atingir até $50 \%{ }^{3}$. O aumento compensatório da frequência cardíaca e a diminuição do volume sistólico podem gerar desequilíbrio entre a oferta e o consumo de oxigênio pelo miocárdio. Esta sobrecarga acarreta impacto na doença isquêmica e na insuficiência cardíaca eventualmente pré-existentes ${ }^{21}$.

Contribuem também para a elevação da resistência vascular periférica fatores humorais. Observa-se aumento nas concentrações de catecolaminas, hormônios do sistema renina-angiotensina-aldosterona e, principalmente, vasopressina ${ }^{10}$. A oligúria é comum, tanto mais quanto maior for a duração do procedimento e decorre da diminuição do fluxo sanguíneo renal pela compressão mecânica e aumento do hormônio antidiurético ${ }^{22}$.

A estase venosa nos membros inferiores propicia a trombose venosa profunda, o que pode ser minimizado através da profilaxia com anticoagulantes, o uso de meias elásticas ou enfaixamento das pernas antes da instalação do pneumoperitônio ${ }^{4,18}$. A dinâmica pulmonar também é afetada, a elevação do diafragma diminui a capacidade residual funcional e a complacência pulmonar dependendo da posição, do grau de céfalo-declive do paciente e da pressão intra-abdominal podendo reduzir-se à metade do normal. As regiões basais pulmonares, comprimidas pela elevação diafragmática devido ao pneumoperitônio, podem apresentar atelectasias que aumentam o espaço morto e desequilibram a relação ventilação/perfusão ocasionando shunt e aumentando a pressão arterial em $\mathrm{CO}^{2}\left(\mathrm{PaCO}^{2} .\right)^{23}$.

Como o pneumoperitônio, via de regra, é mantido com $\mathrm{CO}^{2}$, gás escolhido por ser altamente solúvel, minimizando o risco de embolia gasosa, há aumento da $\mathrm{PaCO}^{2}$ também pela absorção podendo ocasionar acidose respiratória ${ }^{11}$. Na tentativa de diminuir a hipercapnia deve-se aumentar os parâmetros ventilatórios inclusive a pressão de ventilação. Pressões intrapulmonares muito elevadas, maiores que 40 $\mathrm{mmHg}$, aumentam a pressão no território da artéria pulmonar, diminuindo o enchimento do átrio e ventrículo esquerdos afetando ainda mais a pré-carga. É recomendável que se use ventiladores que proporcionem a modalidade de ventilação por pressão controlada em razão do risco de barotrauma. Pelo mesmo motivo deve-se verificar se não há intubação seletiva, que pode acontecer depois da instalação do pneumoperitônio em virtude da elevação da cúpula diafragmática. A pressão positiva expiratória final (PEEP) pode ajudar na ventilação dos alvéolos colabados pelas alterações regionais intra-pulmonares ${ }^{26}$. O gradiente alvéolo-arterial fisiológico de aproximadamente $4 \mathrm{mmHg}$ de $\mathrm{CO}^{2}$ se altera com o pneu-

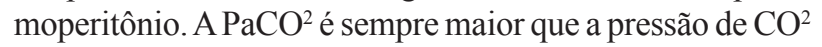
medida pela capnometria em fim de expiração $\left(\mathrm{PETCO}^{2}\right)$, porém não há correlação fixa.

A absorção de $\mathrm{CO}^{2}$ do pneumoperitônio não é linear, é grande nos primeiros 30 minutos tendendo a se estabilizar com o tempo. Medidas gasométricas em procedimentos mais longos podem ser necessárias ${ }^{5}$. A ocorrência de pneumotórax pode ser devida à capacidade do gás de dissecar os tecidos cirurgicamente traumatizados ou com defeitos anatômicos, mas raramente pode ocorrer pneumomediastino. Normalmente estes se resolvem sem necessidade de drenagem, apenas desfazendo o pneumoperitônio. O enfisema celular subcutâneo é comum, mas de resolução espontânea e rápida ${ }^{25}$. A embolia gasosa é rara, porém grave e requer tratamento imediato, cessando a insuflação de $\mathrm{CO}^{2}$, colocando-se o paciente em céfalo-declive, decúbito lateral esquerdo e finalmente, se possível, aspirando o gás com cateter venoso central ou cateter de artéria pulmonar ${ }^{6}$. Em neonatos e lactentes pode haver reabertura do forame oval e do canal arterial com shunt direita-esquerda causando insuficiência cardíaca e risco de embolia gasosa paradoxal ${ }^{1}$.

Lactentes cardiopatas, cuja circulação sistêmica é ducto dependente, como na coartação de aorta, estenose aórtica crítica ou hipoplasia do ventrículo esquerdo e em lactentes com a circulação pulmonar ducto dependente, como na atresia pulmonar e tetralogia de Fallot 
grave, é recomendável que haja correção da cardiopatia antes de procedimento laparoscópico ${ }^{24}$. Há aumento da pressão intracraniana, não relacionada com o aumento da $\mathrm{PaCO}^{2}$, mas proporcional ao aumento da pressão intra-abdominal.

Em pacientes portadores de derivação ventrículoperitoneal ainda ocorre diminuição da drenagem liquórica pela compressão da via de saída, além da diminuição da drenagem venosa pelo mecanismo de Valsalva, ambos contribuindo para o aumento da pressão intracraniana ${ }^{8}$. O $\mathrm{CO}^{2}$ é armazenado em cilindros sob alta pressão e baixa temperatura. Os insufladores reduzem a pressão, mas a temperatura do gás permanece muito baixa. É importante prevenir a hipotermia, principalmente em crianças e naqueles portadores de anemia falciforme.

Em conclusão, a anestesia deve ser geral com ventilação controlada mecânica, mesmo para procedimentos de curta duração, para melhor controle clínico dos pacientes. A monitorização do $\mathrm{CO}^{2}$ expirado pela capnometria é essencial e obrigatória de acordo com o art. 3o inciso III da Resolução 1802/2006 do Conselho Federal de Medicina. O relaxamento muscular adequado é de fundamental valor para o sucesso da operação, visto que praticamente todas as complicações decorrentes da técnica laparoscópica se correlacionam com o aumento da pressão intra-abdominal. Deve-se ter em mente que nem todas as operações que se pretende realizar por via laparoscópica são exequíveis. A conversão em procedimento aberto sempre tem que ser considerada. Finalmente, a relação risco / benefício, levando-se em conta as vantagens no pós-operátório e as intercorrências que podem acontecer no decorrer da intervenção, devem ser avaliadas caso a caso, considerando as variáveis específicas de cada paciente.

Corrêa CMO, Brandão MJN, Hirata ES, Udelsmann A. Anesthetic considerations in laparoscopic surgery. ABCD Arq Bras Cir Dig 2008;21(3):136-8

ABSTRACT - Introduction - Experimental laparoscopy surgeries date of the beginning of the XX century but only in 1960 they started to be tried in humans. In 1985, in Germany, the first colecistectomy was carried through and since then the technique had large spreading and acceptance. The pneumoperitonium has impact in many systems and such must be considered during the anesthesia. Aim - To carry out a literature review of the physiological changes of interest for the anesthesiologist during the videolaparoscopy. Methods - Bibliographical research was done in available anesthesia textbooks in the library in the Faculty of Medicine of the State University of Campinas and published articles of interest beginning on 1990 in PubMed electronic address http://www.ncbi.nlm.nih.gov/pubmed using the following headings: anesthesia, surgery, laparoscopy. Twenty six articles and a book chapter had been reviewed. Conclusions - Laparoscopy has advantages related to reduction in postoperative pain, early hospital discharge and labor activities. Nevertheless, particular care should be taken by the anesthesiologist in hipovolemic patients and those with cardiovascular diseases. The use of capnometry is imperative.

HEADINGS - Anesthesia. Surgery. Laparoscopy.

\section{REFERÊNCIAS}

1. Litynski GS - Laparoscopy: the early attempts, spothtighting Georg Kelling and Hans Christian Jacobeus. JSLS 1997;1:83-5.

2. Litynski GS - Laparoscopy between the world wars: the barriers to transatlantic exchange. Spothighting Heinz Kalk and John C. Ruddoch. JSLS 1997;1:185-8.

3. Litynski GS - Kurt Semm and the automatic insufflators. JSLS 1998;2: 197-200.

4. Mether L - Historical profile of Kurt Karl Stefhan Semm, born march 231927 in Munich, Germany, resident of Tucson, Arizona, USA since 1996. JSLS 2003; 7:185-8

5. Litynski GS - Erich Mühe and the rejection of laparoscopy cholecystectomy (1985): a surgeon a head of his time. JSLS 1998; 15:341-6.

6. Litynski GS - Profiles in laparoscopy: Mouret, Dubois, and Perissat: the laparoscopic breakthrough in Europe (1987-1988). JSLS 1999;3:163-7

7. NIH Consensus Conference - Gallstones and laparoscopic cholecystectomy. JAMA 1993;269:1018-24.

8. Brull JS - Anesthetic considerations for laparoscopic procedures. ASA Refresher Course 1995; 23:15-28.

9. Himmer JP, Putensen C - Comparason of postoperative function after laparoscopy on open laparotomy for cholecystectomy. Anesthesiology 1992;77:675-80.

10. Ortega AE, Richman MF, Hernandez M, Anthoane GJ, Azen S et al. - Inferior vena caval blood flow and cardiac hemodynamics during carbon dioxide pneumoperitoneum. Surg Endosc 1996;10:920-24.

11. Gueugniaud PY, Abisseror M et al. - The hemodynamic effects of pneumoperitoneum during laparoscopic surgery in infants: assessment by continuos esofhageal aortic echo-doppler. Anaesth Analg 1998; 86:260-3.

12. Wedgewood J, Doyle AE - Anaesthesia and laparoscopic surgery in children. Paed Anesth 2001;11:391-9.

13. Cunningham AJ, Brull S - Laparoscopic cholecystectomy; anesthesic implications. Anaesth Analg 1993;76:1120-33.

14. Popken F, Küchle R, Heintz A et al - Cholecystectomy in high risk patients. A compararison between conventional and laparoscopic procedures. Chirurg 1998; 69:1:61-5.

15. Hirvonen EA, Nuutinen LS, Vuolteenaho O - Hormonal responses and cardiac filing pressures in head-up or head-down position pneumoperitoneum in patients undergoing operative laparoscopy. Br J Anaesth 1997;78:128-133.
16. Razvi HA, Fields D, Vargas JC, Vaughan Jr ED, Vukasin A, Sosa RE - Oligúria during laparoscopic surgery: evidence for direct renal compression as an etiologic factor. J Endurol 1996;10:1-4.

17. Morrison CA, Schereiber MA, Olsen SB, Heetz SP, Acosta MM - Femoral venous flow dynamics during intraperitoneal and preperitoneal laparoscopic insufflations. Surg Endoscop 1998;12:1213-16.

18. Emeljanov SJ, Fedenko W, Levite EM, Panfelov SA, Bobrinskaya IG, Fedorov AV et al - Pneumoperitoneum risk prognosis and correction of venous circulation disturbances in laparoscopic surgery. A pilot study. Surg Endosc 1998;12:1224-31.

19. Snabes MC, Poindexter NA - Laparoscopic tubal sterilization under local anesthesia in women with cyanotic disease. Obstet Gynecol 1991;78: 430-40.

20. Koivussalo AM, Lindgren L - Effects of carbon dioxide pneumoperitoneum for laparoscopic cholecystectomy. Acta Anaesthesiol Scand 2000;44:834-41.

21. Walba R, Manazza P - Ventilatory requirements during laparoscopic cholecystectomy. Can J Anaesthesia 1993;40:206-10.

22. Ganem EM - Anestesia para laparoscopia. Em: Ferez D, Vane LA, Posso IP, Potério GMB, Torres MLA - Atualização em Anestesiologia SAESP, São Paulo, Office Editora e Publicidade Ltda., 2004; vol. IX: 120-34.

23. Wahba RW, Tessler MJ, Kleiman SJ - Acute ventilator complications during laparoscopic upper abdominal surgery. Can J Anaesth 1996; 43:77-83.

24. Gonnar C, Fernandez C, Vilalonga A, Nalda MA - Carbon dioxide embolism during laparoscopy and hysteroscopy. Ann Fr Anesth Reanim 1985; 4:380-2.

25. Baroncini S, Gentili A, Pigna A, et al. Anaesthesia for laparoscopic surgery in paediatrics. Minerva Anestesiol 2002;68:406-13.

26. Van der Zee DC, Ba KNMA, Sreeran N et al. - Minimal access surgery in neonates with cardiac anomalies. Ped Endosurg Inn Tech 2003;7:233-8

27. Havelson AL, Barret WL, Iglesias AR, Lee WT, Gaber Sm, Sackier JM - Decreased cerebrospinal fluid absorption during abdominal insufflations. Surg Endoscop. 1998;12:1213-16

Fonte de financiamento: não há Conflito de interesse: não há Recebido para publicação: 23/04/2008 Aceito para publicação: 30/06/2008 